\title{
Predicting the prognostic factors to determine which procedure is the first either laparoscopy or ERCP in cholecystocholedocholithiasis
}

\author{
Awny A El-Zeftawy, MD\#; Mohamed Al-Sayed, $M D^{\# \text {; }}$ \\ Mansour M Morsy, $M D^{*}$; Wael Shelfa, $M D^{*}$ \\ Department of General Surgery, Zagazig University.
}

\begin{abstract}
Background: The obvious goal of therapy in choledocholithiasis is to achieve ductal clearance with the fewest number of interventions, lowest cost and least morbidity. Until now no agreement about the ideal approach for management of stone common bile duct associated with gall bladder stone that may be achieved currently according to a two-step-protocol (endoscopic sphincterotomy associated with laparoscopic cholecystectomy) or by a one-step laparoscopic procedure, including exploration of the CBD and cholecystectomy. The aim of this study is to evaluate both procedures and to determine the prognostic factors to prefer either of both.

Patients and methods: A prospective study included 44 patients treated for gallbladder/CBD stones in the period from October 2008 through December 2010 were divided into two groups, group A treated with Lap first (by the first two authors\#) and group B treated with ERCP first (was treated with the second two authors*).

The obtained results were compared with each other; the mean follow-up period was $6 \pm 1.3$ months (range; 4-8 months).

Results: Total operative time was shorter for $L C+L C B D E$. The incidence of postoperative complications such as cholangitis and pancreatitis are common in group $B$ versus group $A$. Regarding the success rate and conversion rate; in group A two cases were converted to open surgery and one case to ERCP, versus two cases in group $B$ (due to difficult cannulation, so they were converted to lap surgery) and one case due to impacted large stone more than $2 \mathrm{~cm}$ that was converted to open surgery. Late complications are bile leakage in group A, versus three cases in group $B$ in the form of mild pancreatitis and cholangitis and bleeding.

Conclusions: $L C+L C B D E$ and $E R C P / S+L C$ were highly effective in detecting and removing common bile duct stones and were equivalent in overall cost and patient acceptance. LF approach to gallbladder/CBD stones is safe and feasible. It may allow the majority of surgeons to avoid excessively difficult/dangerous surgical procedures as well as unnecessary ERCPs in most cases.

Key words: CBD stones, endoscopy, ERCP.
\end{abstract}

\section{Introduction:}

More than $10 \%$ of the patients with gall bladder stones have synchronous common bile duct stone. ${ }^{1}$ These, according to European Association for Endoscopic Surgery (EAES) guidelines, ${ }^{2}$ should be treated even if asymptomatic. Bile duct stones are found in $7-20 \%$ of patients with symptomatic gallstones. The presence of common bile duct stones significantly increases the morbidity, mortality, and costs of patients with gallstones. ${ }^{1}$

Traditional surgical treatment comprises intraoperative cholangiography to detect the presence of bile duct calculi followed by choledocholithotomy and T-tube placement. For many years this procedure offered effective therapy and was associated with a morbidity rate of $10-15 \%$, a mortality rate of $<1 \%$ (in 
patients under 65 years) and a retained stone rate below $6 \% .2,3$ It was reported that one stage operations have some benefits, as compared to two stage operations. Morbidity after onestage operations was only $7.5 \%$ ( 2 times lower).

Postponing laparoscopic cholecystectomy, after ERCP, makes it difficult to be performed due to the possibility of adhesions at the area of CalotÅfs triangle, this is in additional to the risk of second time anesthesia. ${ }^{4}$ There is now equipoise as to whether surgical treatment alone (open or laparoscopic) or a combined endoscopic-surgical approach is the more effective treatment strategy for the management of choledocholithiasis in association with gallbladder stones. Furthermore, if endoscopic treatment is used in conjunction with surgery, it better to operate before or after endoscopy? Several randomized controlled trials have been published in an attempt to answer one or other, or both, of the above questions.

In 1980s, endoscopic retrograde cholangiopancreatography (ERCP) followed by sphincterotomy has become the most widely used method for imaging and treating CBD stones $^{5,6}$, but recently and in large series ERCP had carried some morbidity (5 to $9.8 \%$ ) and mortality rate $(0.3$ to $2.3 \%),{ }^{7-10}$ due to pancreatitis, duodenal perforation and bleeding procedures. The purpose of our paper is to present our experience with laparoscopic cholecystectomy LC+LCBDE versus $\mathrm{ERCP} / \mathrm{S}+\mathrm{LC}$ (endoscopic sphincterotomy and common duct stone extraction with laparoscopic cholecystectomy).

\section{Patients and methods:}

This is a prospective study that was performed to determine the postoperative outcome of patients treated for CBD stones from January 2009 to December 2010, in two patients groups each with 22 patients. Group A was treated with laparoscopy first and group B treated with endoscopy first. No significant difference was present between both groups regarding age, sex, associated diseases and parameters of inclusion criteria. Inclusion criteria: Age $>18$ years, biliary-type pain, ultrasonographic demonstration of cholecystolithiasis, platelet count $>100,000$ per $\mathrm{mm}^{3}$ and prothrombin time $<3$ seconds of control, American Society of Anesthesiology (ASA) risk grade I or II, common bile duct diameter greater than $6 \mathrm{~mm}$ by ultrasound or computed tomography (CT) scan, intrahepatic duct dilation as determined by ultrasound or CT scan, serum bilirubin greater than $2 \mathrm{mg} / \mathrm{dl}$, alkaline phosphatase and/or lipase more than 1.5 times upper limit of normal within 48 hours of intended first. Exclusion criteria: History of bleeding disorders, platelet count $<100,000$ per $\mathrm{mm}^{3}$ and/or prothrombin time $>3$ seconds over control, uremia as evidenced by a creatinine $>3 \mathrm{mg} / \mathrm{dl}$ and/or blood urea nitrogen $>50 \mathrm{mg} / \mathrm{dl}$, ultrasonography or CT evidence of cirrhosis, liver mass or abscess, or periampullary neoplasm. All patients received preoperative intramuscular injection of vitamin $\mathrm{K}$ (Konakion ${ }^{\circledR}$ ) $10 \mathrm{mg}$ twice daily for 3 days for patients with slightly prolonged PT, and prophylactic broad spectrum antibiotic cefobid ${ }^{\circledR}$ (cefoperazone sodium) as it covers common biliary flora.

\section{The procedure:}

Lap first Group A: LC + LCBDE were performed in a routine. Cholangiograms by antegrade contrast flushing through the cystic duct Figures(1,2,3). During intraoperative cholangiography we used the iv grey cannula sheath instead of a fifth trocar through which we passed the cholangiographic catheter and this made the patient avoid unnecessary fifth trocar in case of negative IOC, and we used the (3Fr) Fogarty catheter after cutting its balloon (this made its guide wire 2-3 cm longer than the catheter itself) as a cholangiocatheter and it was more helpful as its lumen resisted occlusion on exerting external pressure also it has a guide wire. When stones were detected or suspected by cholangiography, trans-cystic exploration was undertaken by balloon or basket. A completion cholangiogram was obtained to confirm that all stones were removed. Once the LCBDE was completed, the cystic duct was ligated and the gallbladder removed or choledochotomy may be done and closed over $\mathrm{T}$ tube or with primary closure Figures(4,5).

ERCP first group B: Patients randomized to $\mathrm{ERCP} / \mathrm{S}+\mathrm{LC}$ were scheduled to undergo the endoscopic procedure using fluoroscopy 
(OEC Diasonics 9400) in the endoscopy suite under moderate sedation (principally intravenous midazolam and meperidine) prior to the intended laparoscopy. If choledocholithiasis was detected or suspected at the time of ERCP, a sphincterotomy was undertaken so that gallstones could be extracted using a balloon catheter or retrieval basket. Small bowel gas was aspirated endoscopically as much as possible at the conclusion of the ERCP. The laparoscopic cholecystectomy was subsequently done Figures(4,5).

\section{Results:}

The most common complaint was right upper quadrant (RUQ) and biliary pain which was found in 18 patients $(81.8 \%)$ in Lap first group, 14 patients $(63.4 \%)$ in ERCP first group, followed by jaundice in 11 patients in the $1^{\text {st }}$ group $(50 \%)$ and in 13 patients $(59.4 \%)$ in the $2^{\text {nd }}$ group Table(1). Abdominal ultrasonography (U/S) was done for all patients in this study. It was able to reveal chronic calcular cholecystitis in all patients. Dilatation of CBD with stone/stones inside was detectable in 33 patients only (75\%). The remaining 11 cases were subjected to MRCP to ensure the diagnosis of calcular obstructive jaundice Figures $(6,7,8)$.

Table (1): Preoperative patient's characteristics.

\begin{tabular}{|l|c|c|c|}
\hline & Group A (Lap. First) & Group B (ERCP) & P value \\
\hline Age 20 to 40 & $11(50 \%)$ & $10(36.4 \%)$ & Ns \\
40 to 60 & $7(31.5 \%)$ & $8(36.4 \%)$ & \\
$>60$ & $4(45.5 \%)$ & $4(45.5 \%)$ & \\
\hline Sex M/F & $16 / 6$ & $17 / 5$ & \\
\hline Symptoms & $18(81.8 \%)$ & $14(63.4 \%)$ & Ns \\
Biliary pain (RUQ) & $11(50 \%)$ & $13(59.4 \%)$ & \\
Jaundice & $9(40.4 \%)$ & $8(36.4 \%)$ & \\
Cholecystitis & $10(44.4 \%)$ & $9(40.4 \%)$ & \\
Pruritis & & & \\
Stone CBD diameter & $7(31.5 \%)$ & $8(36.4 \%)$ & \\
$<15 m m$ & $15(67.5 \%)$ & $14(63.4 \%)$ & \\
$>15 m m$ & $17(77.2 \%)$ & $16(72.7 \%)$ & \\
Ultrasonic sensitivity & $5(22.7 \%)$ & $6(27.2 \%)$ & \\
MRCP & $22 / 22$ & $22 / 22$ & \\
Both & & & \\
\hline
\end{tabular}


Table (2): Postoperative patient's characteristics.

\begin{tabular}{|l|c|c|c|}
\hline & Lap first (22 patients) & ERCP first (22 patients) & P value \\
\hline Operative time & $155-175(165 \pm 1.1)$ & $160-195(180 \pm 2.1)$ & Ns \\
Conversion rate & $3(13.5 \%)$ & $3(13.5 \%)$ & \\
To open & $1(9 \%)$ & $1(4.5 \%)$ & \\
To ERCP & $1(4.5 \%)$ & $2(9 \%)$ & \\
To Lap & & & Ns \\
\hline Intraoperative comp. & $2(9 \%)$ & $3(13.5 \%)$ & \\
Difficult procedure & 1 & $3(13.5 \%)$ & s \\
Late complications & $1(4.5 \%)$ & $3(13.5 \%)$ & \\
\hline Postoperative comp. & $1(4.5 \%)$ & $1(4.5 \%)$ & Ns \\
Persistent bile leak & 0 & $1(4.5 \%)$ & \\
Cholangitis & 0 & $1(4.5 \%)$ & \\
Pancreatitis & 0 & $2-15(5.3)$ & \\
Bleeding & $2-11(3.5)$ & 2443 to 4139 & \\
\hline Hospital stay & 1223 to 4423 & $2948 \pm 32.4]$ & \\
\hline Total Cost & $2637 \pm 12.3]$ & & \\
& & & \\
\hline
\end{tabular}

The total operative time was shorter for LC+LCBDE. No significant difference was present between both groups regarding the morbidity rate. Two cases were converted to open surgery and one to ERCP in group A versus two cases in group B with difficult cannulation which were converted to open surgery and one case due to impacted large stone more than $1 \mathrm{~cm}$. Late complications occurred in group $\mathrm{A}$ in the form of bile leakage in one patient, versus 3 in group B; mild pancreatitis, cholangitis and bleeding.

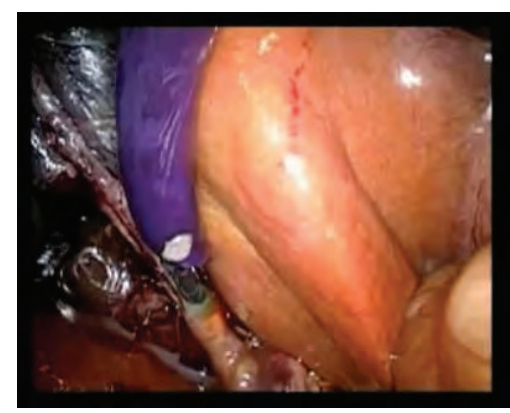

Figure (1): Laparoscopic intra-operative cholangiogram.

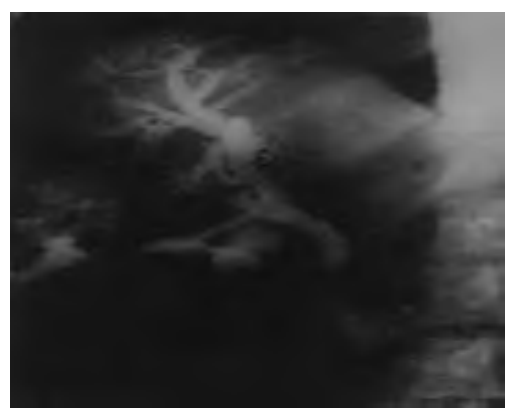

Figure (2): Intra-operative cholangiogram through the cystic duct.

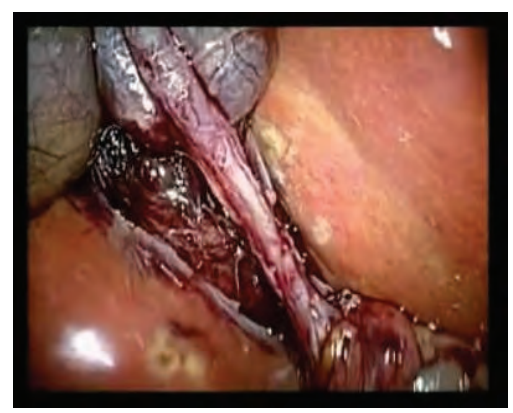

Figure (3): Skeletonization of $C B D$. 


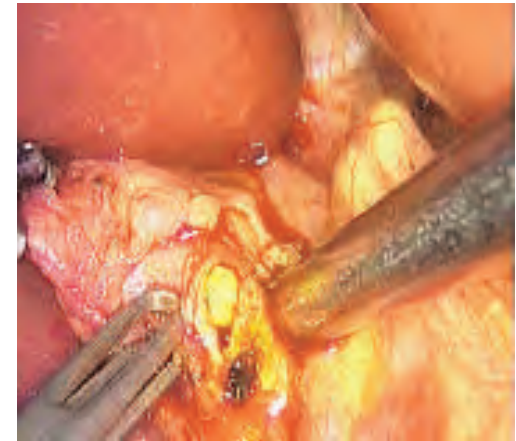

Figure (4): Choledochoctomy incision.

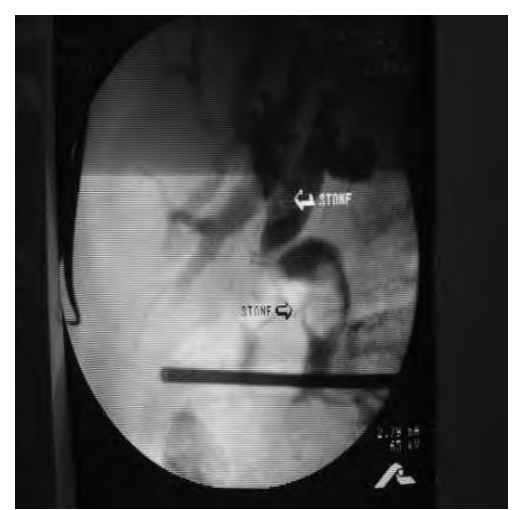

Figure (7): ERCP showing 2 stones in $C B D$.

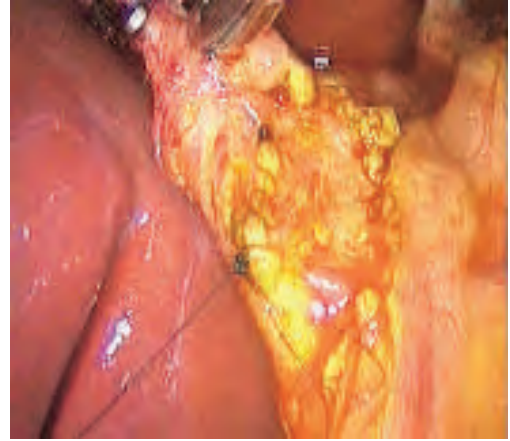

Figure (5): Primary CBD closure.

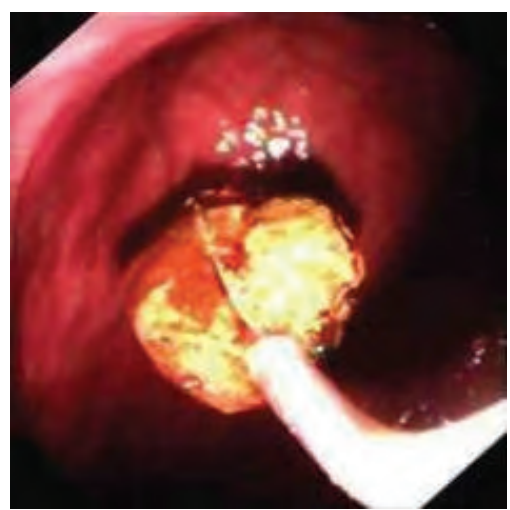

Figure (8): Endoscopic stone extraction by Dormia basket.

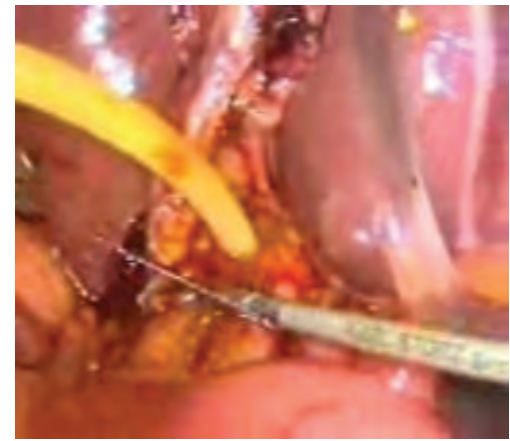

Figure (6): Other case with T tube after $C B D$ incision.

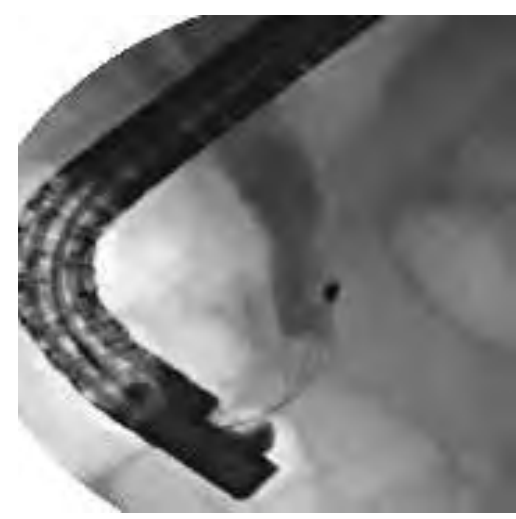

Figure (9): ERCP and stone extraction.
The postoperative hospital stay was shorter for the Lap first group 2 to 11 (mean SD, .5) days vs 2 to 15 [5.3] days in ERCP first; $\mathrm{P}>0.05$. There was no significant difference regarding the conversion rate, three cases were converted to the other procedure or open surgery in both groups.

The postoperative hospital stay was shorter for the Lap first group (2 to 11 days with SD [3.5] days) vs 2 to 15 [5.3] days in ERCP first; $\mathrm{P}>0.05$. There was no significant difference regarding the conversion rate; three cases were converted to the other procedure or open surgery in both groups.

The total cost for LC+LCBDE was not significantly different than those for ERCP/S+LC; 1223 to 4423 [2637 \pm 12.3$]$ in Lap first group vs 2443 to 4139 [2948 \pm 32.4 ] in ERCP first group.

There were no significant differences in both groups regarding the conversion rate and operative complications $3 / 22(13.5 \%)$. The conversion includes one case in whom CBD clearance could not be achieved due to impacted large stone in its lower part and this cases was completed with ERCP, and one case was converted to open approach due to sever adhesions in the Callot's triangle and bleeding, the third case was operated on later after one week due to bile leakage in patients with primary closure of the CBD. In group $\mathrm{B}, 3$ cases had failed procedure two of them due to failed cannulation and were converted to Lap. The third case was converted to open surgery also due to impacted large stone $(15 \mathrm{~mm})$.

Regarding the late postoperative complications we had one case (4.5\%) in group A with persistent bile leak versus three cases in group B (13.5\%); one pancreatitis, one case with cholangitis and one case with bleeding. All were controlled conservatively. The procedure time ranged from 155 to 175 minutes with a mean of $165 \pm 1.1$ minutes in group A versus 195 minutes with a mean of $180 \pm 2.1$ minutes in group B.

\section{Discussion:}

The current options available for the management of choledocholithiasis at the time of LC, include pre-operative ERCP and ES, 
intra-operative ERCP, post-operative ERCP, laparoscopic transcystic common bile duct exploration (LTCCBDE), laparoscopic choledochotomy and LCBDE and open bile duct exploration. ${ }^{3,7}$

The sex and age distribution in our study is in agreement with many studies which found that the gallstones and CBD stones were more common in females and fifth to sixth decades. The most common complaint in our patients was right upper quadrant pain (RUQP) $(72.3 \%)$ and was followed by jaundice (54.5\%). ${ }^{4,5}$ These findings were in some agreement with several studies. ${ }^{4,5}$

MRCP was used successfully to diagnose CBD stones in 11 patients who had equivocal results on transabdominal ultrasonography, these findings were also reported by several studies which found that MRCP has an excellent overall sensitivity of $95 \%$ and a specificity of $97 \%$ for demonstrating CBD stones with no significant differences between it and EUS (Endoscopic Ultrasonography) in the detection of CBD stones. $6,7,8$

Whether to start with ERCP or LC is a matter of debate. We preferred to start with Lap and go on for laparoscopic exploration of the CBD and stone extraction that if failed we will go to the other procedures which may be ERCP or open procedure to choose either of both, depended on the adhesions around Callot's area and the diameter of the stone.

Endoscopic stone retrieval via the ampulla was done after an adequate sphincterotomy (we did not use balloon dilatation) for fear of post ERCP pancreatitis. Other studies denoted that balloon dilatation of the papilla can be an alternative to biliary sphincterotomy, and has been advocated in patients with coagulopathy or cirrhosis, where risk of post sphincterotomy haemorrhage is increased. However, risk of severe PEP is increased in comparison to bleeding, and in the majority of patients undergoing stone extraction balloon dilation should be avoided. 8,9

We used balloon for extraction of common bile duct stones that were $<1 \mathrm{~cm}$ in diameter (to avoid stone impaction). Also we used the basket for extraction of larger stones. This coupled with what was stated by some authors.

Regarding the conversion rate for the other procedure or for open surgery we had only three cases converted in group A (13.5\%) who were converted to open procedure; one due to impacted stone that failed to be extracted and the other case due to bleeding and severe adhesions versus three cases $(13.5 \%)$ in group B two cases were converted due to failed cannulation and were converted to Lap, the third case was converted to open procedure due to impacted stone in the lower part of the common bile duct with no significant difference with an overall success rate $87.5 \% \mathrm{ERCP} / \mathrm{S}+\mathrm{LC}$ versus $91 \%$ in Lap first group. However other studies denoted that the overall success rate of $\mathrm{ERCP} / \mathrm{S}+\mathrm{LC}$ in experienced hands is well established at about $95 \% .{ }^{10}$

Postoperative complications occurred in three patients $(13.5 \%)$ and were minor complications in the form of minimal biliary leakage and were managed conservatively except one that needed conversion to open procedure and mortality was zero. This was in agreement with several studies which reported morbidity rate of $4-16 \%$ and a mortality rate of around $0 \%-2 \% .9,10$ Some studies reported $5.55 \%$ morbidity and mortality rate of zero, this was in agreement with our study. ${ }^{7,11}$

The postoperative cholangitis occurred in group B in form of low-grade fever, nausea, vomiting, epigastric discomfort, diarrhea and one case with pancreatitis versus 0 patients in group A, most probably due to damaged sphincter in group B versus intact sphincter in group A, whereas in other studies symptoms of low-grade cholangitis were detected in $20.36 \%$ patients. ${ }^{9}-13$ Hong et al., stated that EST may cause Oddi's sphincter dysfunction and still is controversial whether it leads to stone recurrence and biliary carcinoma caused by permanent duodenal and pancreatic fluid reflux. ${ }^{14}$

In early cases time of the procedures was longer than in late cases. Our procedure mean time was $165 \pm 1.1$ versus $180 \pm 2.1$ minutes in both groups respectively. This was in agreement with the study carried out by others which denoted mean operative time of 182 minutes. ${ }^{14}$ This was in disagreement with another study which denoted mean operative time of 140.32 minutes. ${ }^{15}$

Postoperative lengths of hospital stay ranged 
from 2 to 11 days (mean 3.5 days) versus 215 (5.3) in both groups respectively. This coupe with what was found by Stanley et al., in their study in which the mean postoperative period was 4.1 days. ${ }^{14,15}$

In our study, there was no significant difference in hospital charges in both procedures. This was in contrast with the study carried out by Hawashi et al which denoted more charges for the procedure in group $\mathrm{B}$ versus A. ${ }^{15,16}$

During intraoperative cholangiography we faced some difficulties in cystic duct cannulation in two cases and we overcome this by using the guide wire of our Fogarty catheter.

Other difficulty was in interpreting the cholangiogram in relation to the appearance of round lucencies (air bubbles vs small stones). To overcome this we slowly injected 2 to 3 $\mathrm{mL}$ of contrast under constant fluoroscopic control then we withdrew the plunger to create a vacuum. We repeated this maneuver twice and observed the motion and appearance of the lucency. Bubbles move in synchrony with the column of contrast. Stones tend to adhere to the wall of the duct and do not move. This problem was faced and solved in the same way by Stanley et al, at 2010.17

The procedures were completed in 20 cases (91\%) with two cases converted to open surgery, one was due to severe adhesions in Calot's triangle and one case because of stone removal failure (stone removed later on by ERCP). Similar study carried out by Hong al, denoted rate of $80 \%$ to $99 \% .{ }^{18,19}$ In other studies success rate of $80 \%$ to $95 \%$ were reported. ${ }^{19,20}$

Ten patients were subjected to choledochotomy in whom CBD was closed primarily in 7 cases and the other 3 cases were drained by $\mathrm{T}$ - tube. Biliary leakage occurred postoperatively in one patient of the seven cases who were closed primary. Many studies comparing primary closure versus T-tube drainage suggest similar rates of complications with shorter operating times and a trend toward shorter hospital stays with primary closure. But some authors like Roush et al and Soe et al believed that T-tube drainage is preferred for the safety of the patient. ${ }^{18-20}$

In this study we used the choledochoscope for 3 patients and it was helpful to confirm bile duct clearance and to visualize the proximal portion of the CBD but in these cases the operating time was a little longer. In the other five cases choledochoscope was replaced (due to some technical problems in the choledochoscope) by fluoroscopic guidance and confirmatory IOC. This was in agreement with Rhodes et al who denoted nearly similar efficacy between fluoroscopic guidance. ${ }^{21,22}$ This was in contrary to Sgourakis et al who reported that the use of a flexible choledochoscope is preferable tfluoroscopic guidance. $^{22}$

\section{Conclusions:}

Both $\mathrm{ERCP} / \mathrm{S}+\mathrm{LC}$ and $\mathrm{LC}+\mathrm{LCBDE}$ were highly effective detecting and removing common bile duct stones and were equivalent patient acceptance. However, the overall of hospitalization was shorter and physician fees lower LC+LCBDE.

\section{References:}

1- Martin DJ, Vernon DR, Toouli J: Surgical versus endoscopic treatment of bile duct stones. Cochrane Database Syst Rev 2006; (2): CD003327.

2- Morino M, Baracchi F, Miglietta C, Furlan N, Ragona R, Garbarini A: Preoperative endoscopic sphincterotomy versus laparoendoscopic rendezvous in patients with gallbladder and bile duct stones. Ann Surg 2006; 244: 889-893.

3- Clayton ES, Connor S, Alexakis N, Leandros E: Meta-analysis of endoscopy and surgery versus surgery alone for common bile duct stones with the gallbladder in situ. $\mathrm{Br} J$ Surg 2006; 93:1185-1191.

4- Barwood NT, Valinsky LJ, Hobbs MS, Fletcher DR, Knuiman MW, Ridout SC: Changing methods of imaging the common bile duct in the laparoscopic cholecystectomy era in Western Australia: Implications for surgical practice. Ann Surg 2002; 235: 41-50.

5- Ghazi A, McSherry CK: Endoscopic retrograde cholangiopancreatography and sphincterotomy. Ann Surg 1984; 199: 2127.

6- Rhodes M, Sussman L, Cohen L, Lewis 
MP: Randomised trial of laparoscopic exploration of common bile duct versus postoperative endoscopic retrograde cholangiography for common bile duct stones. Lancet 1998; 351: 159-161.

7- Sgourakis G, Karaliotas K: Laparoscopic common bile duct exploration and cholecystectomy versus endoscopic stone extraction and laparoscopic cholecystectomy for choledocholithiasis. A prospective randomized study. Minerva Chirurgica 57(4): 467-474.

8- Wojtun S, Gil J, Gietka W, Gil M: Endoscopic sphincterotomy for choledocholithiasis: A prospective singlecenter study on the short-term and longterm treatment results in 483 patients. Endoscopy 1997; 29(4): 258-265.

9- Giurgiu DI, Margulies DR, Carroll BJ, et al: Laparoscopic common bile duct exploration: Long-term outcome. Arch Surg 1999; 134(8): 839-843.

10-Millat B, Deleuze A, Atger J, et al: Treatment of common bile duct lithiasis under laparoscopy: A prospective multicenter study in 189 patients. Gastroenterol Clin Biol 1996; 20(4): 339345.

11-Vaira D, D'Anna L, Ainley C, et al: Endoscopic sphincterotomy in 1000 consecutive patients. Lancet 1989; 2(8660): 431-434.

12-Geenen JE, Vennes JA, Silvis SE: Resume of a seminar on endoscopic retrograde sphincterotomy (ERS). Gastrointest Endosc 1981; 27(1): 31-38.

13-Freeman ML, Nelson DB, Sherman S, et al: Complications of endoscopic biliary sphincterotomy. N Engl J Med 1996; 335(13): 909-918.

14-Phillips EH: Controversies in the management of common duct calculi. Surg Clin North Am 1994; 74(4): 931-948.

15-Hong DF, Xin Y, Chen DW: Comparison of laparoscopic cholecystectomy combined with intraoperative endoscopic sphincterotomy and laparoscopic exploration of the common bile duct for cholecystocholedocholithiasis. Surg Endosc 2006; 20(3): 424-427.

16-Boender J, Nix GA, de Ridder MA, et al: Endoscopic papillotomy for common bile duct stones: Factors influencing the complication rate. Endoscopy 1994; 26(2): 209-216.

17-Hawasli A, Lloyd L, Cacucci B: Management of choledocholithiasis in the era of laparoscopic surgery. Am Surg 2000; 66(5): 425-430.

18-Stanley J, Cello JP, Horn JK, Siperstein AE, William P, Campbell AR, Mackersie RC, Alex, CCRC, Kreuwel HTC, Harris HW: MD Prospective randomized trial of $\mathrm{LC}+\mathrm{LCBDE}$ vs ERCP/S+LC for common bile duct stone. Disease Arch Surg 2010; 145(1): 28-33.

19-Liberman MA, Phillips EH, Carroll BJ, Fallas MJ, Rosenthal R, Hiatt J: Costeffective management of complicated choledocholithiasis: Laparoscopic transcystic duct exploration or endoscopic sphincterotomy. J Am Coll Surg 1996; 182(6): 488-494.

20-Roush TS, Traverso LW: Management and long-term follow-up of patients with positive cholangiograms during laparoscopic cholecystectomy. American Journal of Surgery 169(5): 484-487.

21-Seo DW: Prospective analysis of endoscopic papillary balloon dilatation and endoscopic sphincterotomy for removal of common bile duct stones. Gastrointestinal Endoscopy 52(1): 140-142.

22-Rhodes M, Sussman L, Cohen L, Lewis MP: Randomised trial of laparoscopic exploration of common bile duct versus postoperative endoscopic retrograde cholangiography for common bile duct stones. Lancet 1998; 351(9097): 159-161. 